\title{
Automated User Interface Generation using Generative Adversarial Networks
}

\author{
Vedant Nandoskar \\ Student, \\ Department of Information \\ Technology, \\ Vidyalankar Institute of Technology, \\ Mumbai
}

\author{
Rishi Pandya \\ Student, \\ Department of Information \\ Technology, \\ Vidyalankar Institute of Technology, \\ Mumbai
}

\author{
Devesh Bhangale \\ Student, \\ Department of Information \\ Technology, \\ Vidyalankar Institute of Technology, \\ Mumbai
}

\author{
Ajay Dhruv \\ Assistant Professor, \\ Department of Information Technology, \\ Vidyalankar Institute of Technology, \\ Mumbai
}

\begin{abstract}
A website is the most important marketing tool for a business. Industries are discovering the huge amount of opportunity a digital presence creates. Equally important for a website is a good User Interface because it can make or mar your digital presence and customer base. As a result, people who do not possess the required technical knowledge suffer critical losses due to the lack of a website or a website with substandard UI. The primary objective of this system is to "Enable everyone to create for the web", focusing more on the latter problem. The goal is to design a machine algorithm that always serves a new set of interactive vector-based UI mock-up images based on the user responses to a predefined number of questions. The proposed system can be used in various fields such as creating website designs for businesses, portfolios, online stores, blog sites, etc. Deep learning and GAN models will help the algorithm learn exact user requirements, preferences, and modifications. GAN will also help the system to deliver a set of new design mock-ups every time eliminating the current problem of a predefined number of templates.
\end{abstract}

\section{General Terms}

User Interface system, Automated intelligent system, Deep Learning

\section{Keywords}

Generative Adversarial Networks (GAN), User Interface Design, Automated systems, Intelligent informative agents, Anonymous user profiling, Recommendation systems.

\section{INTRODUCTION}

According to the web credibility research program of Stanford, $75 \%$ of users admit to making judgments about a company's credibility based on their website's design [1][2]. First impressions of people about a business are $94 \%$ designrelated.

Hence, it can be supposed that having a good website design tops the idea of having a website in the first place. Creating good designs is a daunting task, and the problem increases exponentially for people from a non-technical background. The proposed research paper tackles the problem by providing a novel, non-technical, and automated solution by constantly engaging with the question "Can $\mathrm{AI}$ in web development positively impact the creation of remarkable website designs".

The presence of a good UI is essential to positively market a business or a service among the clients, which is not present in most cases. Manually creating web designs produces better quality results only and only if created by experienced designers. The shortcomings of this technique overpower the pros. The alternate methods like WordPress and Wix provide fixed templates and also fail to recognize the lack of coding knowledge in people from non-technical background. Thus, the development of a website for their businesses seems like a far-fetched idea making them rely on third parties where the cost factor and the availability of the designers in the need of the hour come into play. Along with that, sometimes it is redundant to consider professional help for designs that are required for college projects, hackathons, or small-scale personal projects where budgets for UI do not exist as people are more feature-focused than design-focused. Thus, the complexities of creating a good quality website design hinder people to expand their market business and create an online presence, which is extremely vital in modern times.

To overcome the mentioned barriers and provide a feasible solution, 'Automated UI' was created. The system works in a simple manner mainly targeting ease of use, industrial quality designs, customization, and inventiveness. It also eliminates the need for a good UI designer even for large scale projects as the generated designs will match those of experienced designers. A set of machine-generated UI mock-ups will be proposed, giving the user the liberty to choose a mock-up most preferred, and also modify it through drag and drop features. The output set obtained will comprise of vector images. The technological requirement to use the proposed system will be null as the user will just have to answer a predefined set of questions and obtain an output of a mock-up set. The incorporated deep learning techniques and GAN model will ensure that new designs are always put up on the table by constantly monitoring user responses and modifications and suggest different, more favored outputs to the user each time. Hence, all the problems are eliminated in the path of a successful business web design.

Automated UI is a carefully crafted system that takes into 
account the barriers faced by a person in terms of creating a website design. The proposed system is a "NO CODE" application that serves the precise UI for a specific business purpose. It takes into account user requirements received by asking a list of predefined questions. The user is given the power to also customize the preferred output through simple drag and drop tools that not only make the process easy but also engaging. The system consistently delivers new outputs each time which is acquired with the help of Generative Adversarial Network and Deep Learning. Thus, the simplicity of creating a good-looking website UI and achieving a striking online presence will be truly achieved through this system.

\section{LITERATURE SURVEY}

Lu, Y. [10] proposed a solution for the generation of a website by using features of two websites. In a GAN, a generator and discriminator are trained together until the discriminator cannot distinguish the generated instances from the instances in the source domain. After this training is done, the GAN models can be used to combine the features of two websites and create a unique third website out of them. Han Zhang [12] proposed a new training methodology for GANs which is attention-driven, and focuses on long-range dependency modeling for image generation tasks. Recently, it has been seen that generator conditioning affects GAN performance. Leveraging upon this insight, spectral normalization is been applied to the GAN generator and it resulted in improved training dynamics. Yan $\mathrm{Wu}$ [14] introduces a new form of latent optimization inspired by the CS-GAN and shows that it improves adversarial dynamics by enhancing interactions between the discriminator and the generator further demonstrating that latent optimization can significantly improve GAN training. Tero Karras [16] proposed a new architecture that is based on style transfer learning and leads to an automatically learned, unsupervised separation of highlevel attributes and stochastic variation in the generated images and it enables intuitive, scale-specific control of the synthesis. For example, when considering a human face, the high-level attributes are separated i.e., pose and identity and the stochastic variation deals with human freckles, hair, etc. Timo Aila [19] puts forth a style-based GAN architecture that results in data-driven unconditional generative image modeling. This paper presents generator normalization, revisit progressive growing, and attempts to regularize the generator to encourage good conditioning in the mapping from latent vectors to images thus, improving the image quality. Ashutosh Kumar [23] proposed a solution for orders made in ecommerce websites by using a Generative Adversarial Network (GAN). Once trained, the generator in the GAN could generate any number of probable orders. This solution consisted of creating a dense and low-dimensional representation of e-commerce orders, train an ecommerceGAN (ecGAN) with real orders to show the feasibility of the proposed paradigm, and train an ecommerceconditional-GAN (ec2GAN) to generate the plausible orders involving a particular product

\section{PROPOSED SYSTEM}

The 'Automated UI Generation' system is intended to generate UIs based on a user's needs. The architecture of the proposed system is built by a few components each performing a specific task essential to provide the desired results.

The basic workflow of the entire system is that initially, every user will have their profile to which they $\log$ in or sign up.
After logging into the application, the user will answer a questionnaire hinting at the website design it wants the system to create. The questions are based on the product/service the user wants a design for, the color palette to be used, the targeted audience for the product/service, whether animations are required, the layout of components, etc.). The questions are basically a method to engage the naïve user with the system. The responses to these questions would be used to adjust the parameters to the model. The model would then use these responses and also use the user profiling data stored in the database (if the user has used the system before) to draw inferences and provide the best possible results. There would be five results provided to the user, and the user has a choice to choose the mockup (result) which suits him/her best. If the user has used the software in the past, the system automatically recommends a few UI mock-ups.

The system has the following components as its foundational ground for generating mockups.

1. A questionnaire

2. Response analyzer

3. GAN model

Apart from these, the effectiveness of results can be further enhanced by adding in complimentary components such as

1. An anonymously profiling agent for users.

2. A dedicated database for storing previous results.

3. Inference engine to make sense of the stored data.

4. A recommendation system for suggesting tailor-made UIs out of the box.

These components are explained in detail in the sections.

\subsection{Questionnaire}

The questionnaire is the starting point for generating mockups. It forms the basis of understanding the user's needs for the mockups. This step could be done in a lot of ways like integrating a form or asking users to adjust parameters by themselves. An interactive questionnaire is preferred to simplify the task to a naïve and uninitiated user. The questions are based on the product/service the user wants a design for, the color palette to be used, the targeted audience for the product/service, whether animations are required, the layout of components, etc.). The questions are basically a method to engage the naïve user with the system. The responses to these questions would be used to adjust the parameters to the model. The probable questions and the insights they give are highlighted in Table 1 below.

Table 1. Sample questions and the insights they provide

\begin{tabular}{|l|l|l|}
\hline \multicolumn{1}{|c|}{ Question } & \multicolumn{1}{c|}{$\begin{array}{c}\text { Example } \\
\text { responses }\end{array}$} & \multicolumn{1}{c|}{$\begin{array}{c}\text { Insight they } \\
\text { provide }\end{array}$} \\
\hline $\begin{array}{l}\text { What type of } \\
\text { website do you } \\
\text { want? }\end{array}$ & $\begin{array}{l}\text { Portfolio, } \\
\text { Consultancy } \\
\text { Page }\end{array}$ & $\begin{array}{l}\text { Components that } \\
\text { should be present } \\
\text { in a website }\end{array}$ \\
\hline $\begin{array}{l}\text { What color theme } \\
\text { do you need for } \\
\text { the website? }\end{array}$ & $\begin{array}{l}\text { Light, Dark, } \\
\text { Primary } \\
\text { colors }\end{array}$ & $\begin{array}{l}\text { The color scheme } \\
\text { to be used in the } \\
\text { website }\end{array}$ \\
\hline $\begin{array}{l}\text { What kind of } \\
\text { audience is the } \\
\text { website intended } \\
\text { for? }\end{array}$ & $\begin{array}{l}\text { Teenagers, } \\
\text { Professionals, } \\
\text { General }\end{array}$ & $\begin{array}{l}\text { The kind of } \\
\text { features, styling, } \\
\text { fonts, layouts to be } \\
\text { used. }\end{array}$ \\
\hline $\begin{array}{l}\text { Would the website } \\
\text { be used on }\end{array}$ & Yes/No & $\begin{array}{l}\text { How responsive } \\
\text { the website should }\end{array}$ \\
\hline
\end{tabular}




\begin{tabular}{|c|c|}
\hline $\begin{array}{l}\text { handheld or larger } \\
\text { screens? }\end{array}$ & $\begin{array}{l}\text { be? Is it just for } \\
\text { desktops or would } \\
\text { the mockups for } \\
\text { mobile and larger } \\
\text { screens be } \\
\text { provided as well }\end{array}$ \\
\hline
\end{tabular}

\subsection{Response Analyzer, Inference Engine}

This is a pre-programmed system that would be tasked with handling the responses, deriving insights and converting into 'weights' that could be fed into the model to generate appropriate responses. There are two ways to design this analyzer - one being an NLP (Natural Language Processing) based approach and the other approach where the weights are predefined for a set of finite responses. The NLP based approach provides more flexibility in terms of analyzing a wide range of responses, while the predefined weights one provides accuracy and confidence in choosing proper weights. For the proposed system, the predefined weights approach would be more suitable since the responses to the questionnaire would be finite and known beforehand. This allows the system to be accurate and effective in choosing weights while also saving 'wait time' as opposed to analyzing responses using an NLP based system.

\subsection{The GAN model}

Generative Adversarial Networks (GANs) is the primary algorithm that will be used to create a set of new UI mock-ups each time. GANs are an approach to generative modelling using deep learning methods, such as convolutional neural networks [4]. Generative modelling is an unsupervised learning task in machine learning that involves automatically discovering and learning the regularities or patterns in input data in such a way that the model can be used to generate or output new examples that plausibly could have been drawn from the original dataset [4]. For example, a model is trained on a dataset of 1500 social-media mock-up images [3]. The model will provide a new social media mock-up image. The output design generated does not exist for any website but was created after learning the regularities and patterns in the dataset images. The generated output will consist of images, which could be further developed to make them user- customizable and also provide various editor tools. The system would be able to provide five output images based on the responses provided by the user to empower the user with the option of choosing the best output instead of settling strictly on machine-generated results. These choices could also be recorded and stored in the user's profiling database. That could then be used to enhance the results based on their choices for succeeding trials and designs.

\subsection{User Profiling and Database}

The system would have a User Profiling system which would help analyse the behaviours and preferences of every individual user. This system is to be designed in such a way that it protects the anonymity of the users [6]. Such a system has been successfully tested in scenarios where the tasks were information-based, and the intelligent agents needed individual user data to provide the best results [7]

The database to be used is a NoSQL database (for example, MongoDB) because such database systems are proven to be more effective and faster when handling semi-structured data [5]. Since the system would contain a lot of semi-structured data relating to the behaviour and preferences of individual users, a NoSQL database like MongoDB is certainly a better choice.

\subsection{Recommendation System}

The recommendation system would be used to provide automated tailormade UI mock-ups to the user based on his/her previous activity [8]. This system would make use of the data in the database and the inferences made by the inference engine with the help of the response analyser. The recommendation system that most suits this application is Collaborative Filtering based recommender system. This system is effective in cases where a grouping of individuals with similar preferences is possible. Since the users share their preferences to a certain extent, it could be used to recommend similar results to an entire group of people with similar preferences based on the behaviours of a fraction of people from that group. The recommendations would prove to be a strong feature as that would help the user have a wider variety of choices in far less time. 


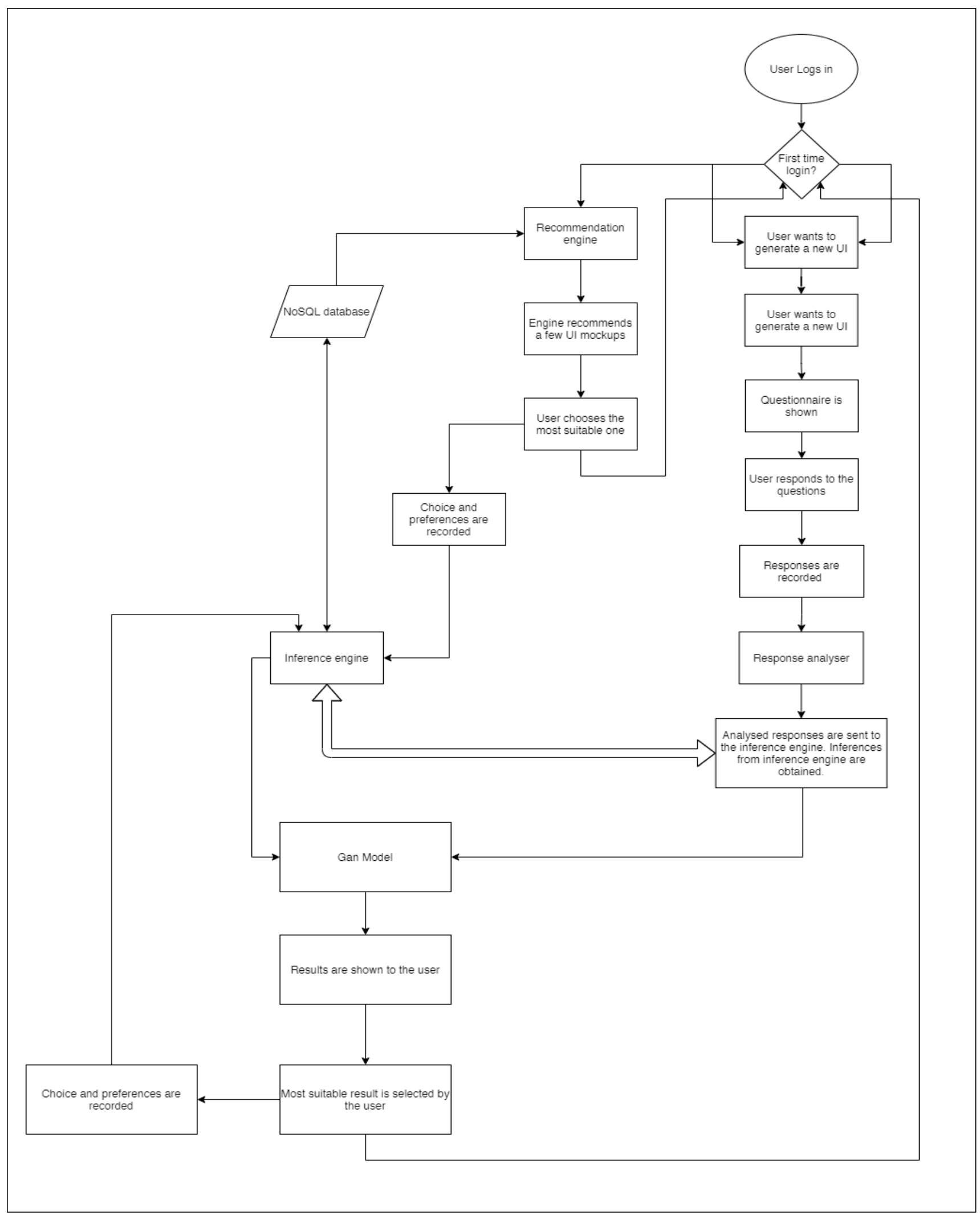

Fig 2: Automated UI Workflow Diagram

\section{EXPECTED RESULTS}

The expected results are obtained in the form of full-sized low-fidelity UI wireframes. These results are wireframes generated from the model. Typically, the proposed system should be delivering multiple results at one go. This allows the user to have the ability to choose the best one for his/her needs, rather than repeating the process in case of an unsatisfactory result. The proposed model will learn the user's choices and use that data to provide more accurate predictions for later trials. This is done by using an additional layer of feedback mechanism over the model's trained layers. This layer is stored in the database as its specific to each user. In 
this way, the results obtained get more precise over use, eventually increasing its accuracy.

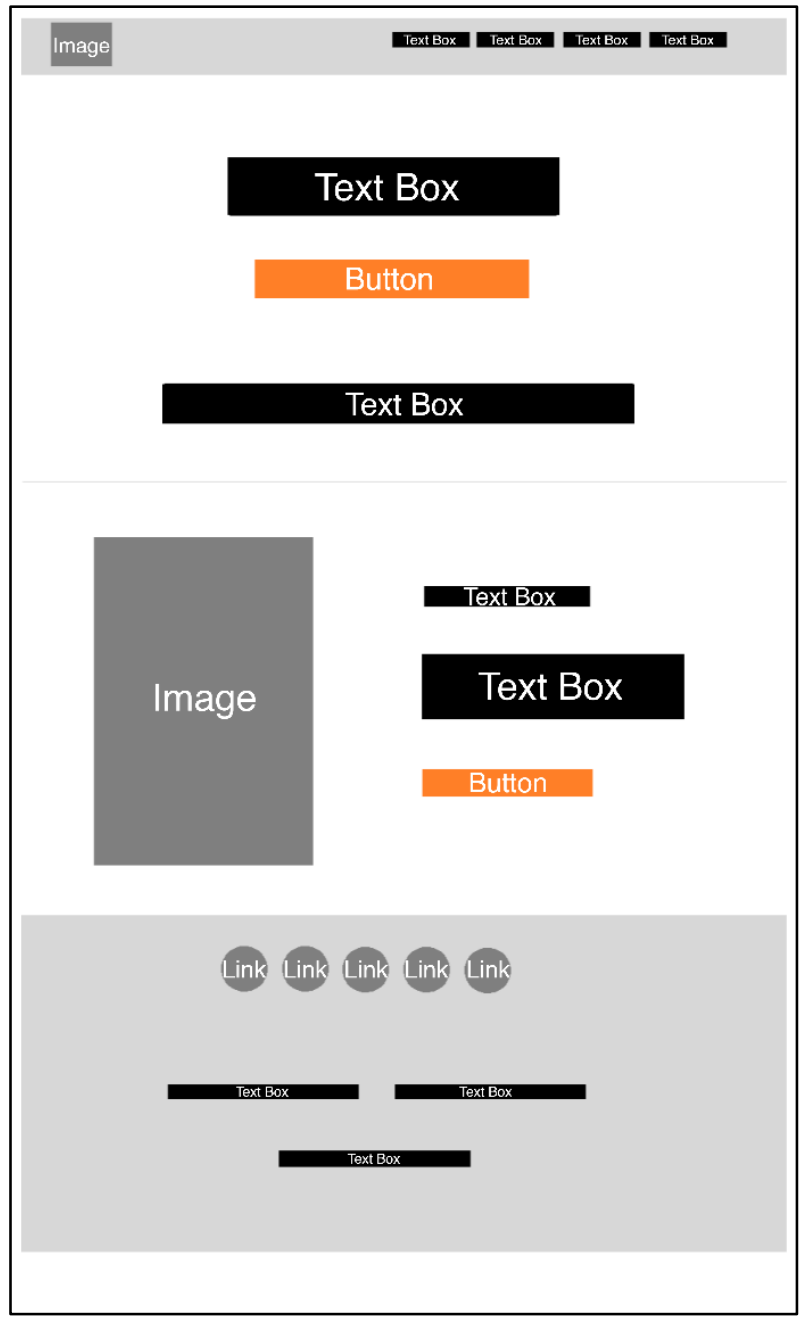

Fig 3: Sample UI Expected Output

The output is colour and shape coded. This makes it easier for the system to detect what type of element exists at that position. The index for coded elements is explained in the following table.

Table 2. Sample coding scheme for UI elements

\begin{tabular}{|l|l|l|}
\hline \multicolumn{1}{|c|}{ Color } & \multicolumn{1}{c|}{ Shape } & \multicolumn{1}{c|}{ UI element } \\
\hline Grey & Quadrilateral & Images \\
\hline Black & Quadrilateral & Textbox \\
\hline Grey & Circle & Link \\
\hline Orange & Quadrilateral & Button \\
\hline
\end{tabular}

\section{CONCLUSION}

Creating and maintaining an online presence in these times is a necessity and can be a challenging task for people who are not technically inclined. The proposed system can thus enable small businesses and individuals to get their unique and personalized online presence in an efficient manner. This system takes into account the various needs of different users and learns to adapt to them, thus, providing better results with every subsequent use. GANs are used to generate unique user interface designs with every individual use, meeting the best possible culmination of existing UI paradigms and user's demands. The editor provided with the system empowers enhanced personalization to completely meet the requirements of the user. Consequently, the proposed system has the potential to find its use in a wide domain of applications.

\section{FUTURE SCOPE}

The foremost future scope will be to expand the domain that "Automated UI" presently aids, blooming out from just website design templates to much more, likely company logos, posters, brochures, newsletters, etc. Another thoughtful feature will be for users to get the ability to share their customized templates publicly. People can follow a person whose design templates they like and can also like/save/use the templates. This will help increase a good design reach, thus helping the GAN model to learn more.

\section{ACKNOWLEDGMENT}

We would like to express our deepest gratitude to our project guide, Prof. Ajay Dhruv, who provided us an opportunity to conduct the research and provided us invaluable guidance throughout this research by sharing his knowledge and expertise.

\section{REFERENCES}

[1] B.J. Fogg, Jonathan Marshall, Alex Osipovich and Chris Varma. "Elements that Affect Web Credibility: Early Results from a Self-Report Study". Proceedings of ACM CHI 2000 Conference on Human Factors in Computing Systems, v.2, New York: ACM Press.

[2] B.J. Fogg and Shawn Tseng. "The Elements of Computer Credibility". Proceedings of ACM CHI 99 Conference on Human Factors in Computing Systems, v.1, pp. 80-87. New York: ACM Press.

[3] Shamsolmoali, P., "Image Synthesis with Adversarial Networks: A Comprehensive Survey and Case Studies", arXiv e-prints, 2020.

[4] Chang, Ming-Li \& Chua, Hui Na. (2018). SQL \& NoSQL Database Comparison: from Performance Perspective in Supporting Semi-Structured Data.

[5] B. S. Atote, S. Zahoor, B. Dangra and M. Bedekar, "Personalization in user profiling: Privacy and security issues," 2016 International Conference on Internet of Things and Applications (IOTA), Pune, India, 2016, pp. 415-417, doi: 10.1109/IOTA.2016.7562763.

[6] Pradeep, Kali \& Bhaskar, M. (2018). Comparative analysis of recommender systems and its enhancements. International Journal of Engineering and Technology. 7. 304-310.

[7] Hauger, Stefan \& Tso, Karen \& Schmidt-Thieme, Lars. (2007). Comparison of Recommender System Algorithms focusing on the New-Item and User-Bias Problem. 525-532. 10.1007/978-3-540-78246-9_62.

[8] M. V. Murali, T. G. Vishnu and N. Victor, "A Collaborative Filtering based Recommender System for Suggesting New Trends in Any Domain of Research," 2019 5th International Conference on Advanced Computing \& Communication Systems (ICACCS), Coimbatore, India, 2019, pp. 550-553, doi: 10.1109/ICACCS.2019.8728409.

[9] Jordi Cabot, "WordPress: A Content Management 
System to Democratize Publishing", IEEE Software vol.35, Issue: 3, May/June 2018.

[10] Lu, Y., \& Velipasalar, S. (2019). Autonomous Choice of Deep Neural Network Parameters by a Modified Generative Adversarial Network. 2019 IEEE International Conference on Image Processing (ICIP).

[11] Vincent Dumoulin, Jonathon Shlens, Manjunath Kudlur, A learned representation for artistic style.

[12] Han Zhang, Ian Goodfellow, Dimitris Metaxas, Augustus Odena, "Self-attention generative adversarial networks", CoRR, 2018

[13] Leon A. Gatys, Alexander S. Ecker, Matthias Bethge, Image Style Transfer Using Convolutional Neural Networks, 2016 IEEE Conference on Computer Vision and Pattern Recognition (CVPR)

[14] Yan Wu, Jeff Donahue, David Balduzzi, Karen Simonyan, Timothy Lillicrap (2019). LOGAN: Latent Optimisation for Generative Adversarial Networks.

[15] Lucas Theis, Aäron van den Oord, and Matthias Bethge, "A note on the evaluation of generative models", arXiv preprint arXiv:1511.01844 (2015)

[16] Tero Karras, Samuli Laine, Timo Aila, A Style-Based Generator Architecture for Generative Adversarial Networks, 2019 IEEE/CVF Conference on Computer Vision and Pattern Recognition (CVPR)

[17] T. Chen, M. Lucie, N. Houlsby and S. Geliy, "On self modulation for generative adversarial networks", CoRR, 2018.

[18] I. Goodfellow, I. Pouget-Abadie, M. Mirza, B. Xu, D. Warde-Farley, S. Ozair, et al., "Generative Adversarial Networks", NIPS, 2014.

[19] Timo Aila, Tero Karras, Samuli Laine, Miika Aittala, Janne Hellsten, Jaakko Lehtinen, "Analyzing and Improving the Image Quality of StyleGAN", CVPR
2020

[20] John Glover, "Modeling documents with Generative Adversarial Networks", arXiv preprint arXiv:1612.09122 (2016).

[21] X. Yao, "Evolving artificial neural networks", Proc. IEEE, vol. 87, no. 9, pp. 1423-1447, Sep. 1999.

[22] Chaoyue Wang, Chang Xu, Xin Yao, Dacheng Tao, "Evolutionary Generative Adversarial Networks", IEEE Transactions on Evolutionary Computation vol.23, Issue: 6, Dec 2019.

[23] Ashutosh Kumar, Arijit Biswas, Subhajit Sanyal, "eCommerceGAN: A Generative Adversarial Network for E-commerce", In Proceedings of, April 2018 (Arxiv).

[24] T. Nguyen, P. Vu, H. Pham and T. Nguyen, "Deep Learning UI Design Patterns of Mobile Apps," 2018 IEEE/ACM 40th International Conference on Software Engineering: New Ideas and Emerging Technologies Results (ICSE-NIER), Gothenburg, Sweden, 2018, pp. 65-68.

[25] Huang, H., Yu, P. S., and Wang, C., "An Introduction to Image Synthesis with Generative Adversarial Nets", arXiv e-prints, 2018.

[26] Ali, Wajid \& Majeed, Muhammad \& Raza, Ali \& Shafique, Muhammad Usman. (2019). Comparison between SQL and NoSQL Databases and Their Relationship with Big Data Analytics. Asian Journal of Computer Science and Information Technology. 4. 1-10. 10.9734/AJRCOS/2019/v4i230108.

[27] Dickinson, Ian \& Reynolds, Dave \& Banks, Dave \& Cayzer, Steve \& Vora, Poorvi. (2003). User Profiling with Privacy: A Framework for Adaptive Information Agents. Lecture Notes in Artificial Intelligence (Subseries of Lecture Notes in Computer Science). 2586. 123-151. 10.1007/3-540-36561-3_6. 